\title{
Development of Vehicle Speed Estimation Technique using Image Processing
}

\author{
Nagaratna M Raikar \\ Department of ISE \\ Ramaiah Institute of Technology \\ Bengaluru, Karnataka INDIA
}

\author{
Dr. Megha P Arakeri \\ Department of ISE, Center of Imaging Technologies \\ Ramaiah Institute of Technology \\ Bengaluru, Karnataka, INDIA
}

\begin{abstract}
In India, the surveillance system and intelligent traffic management is the essential requirement for smart city improvement. But nowadays, the number of accidents on the road rises every year due to more vehicles on the road. Vehicle speed is only one reason for road accidents. So that traffic management authority needs a better surveillance system. Due to the significant expense of radar and less precision, the radar system is not able to become popular in the traffic surveillance system. And the inductive loops technique also needs high support. Therefore it is necessary to propose some mechanisms to overcome such problems. This paper proposed a novel computer vision-based automated system for multi-vehicle detection, tracking, and estimation of vehicle speed in a video sequence by using image processing. This proposed method contains several steps for vehicle speed estimation. In the first step, pre-processing is applied to extracted frames to reduce noise. And then, the gradient edge detection algorithm is used for moving vehicle edge extraction. The next step is template matching by using a normalized cross-correlation method. In this proposed system, detected vehicles tracked by using the vehicle's frame coordinates. Finally, speed is estimated using the number of frames and frame rate. The detection and tracking accuracy achieved by the proposed approach is about $97.33 \%$ and $97.2 \%$. Overall precision, error rate, F1_score, sensitivity, kappa value is $98.19 \%, 0.0267,97.43,96.68 \%$ and 0.9466 respectively.
\end{abstract}

Keywords-Background Subtraction, Gradient Edge Detection, Image Processing, Multi-vehicle Detection, Vehicle Tracking, Vehicle Speed Estimation.

\section{INTRODUCTION}

India is one of the busiest nations in the world in terms of road traffic. The vehicle business over the south Asian country turned into the fourth biggest in the world in 2017. In 2019, there were just about 3 million new car enrollments in the country [1]. The Indian street organizes, crossing more than 5 million kilometers, conveyed right around $90 \%$ of the nation's traveler traffic and around $65 \%$ of the products. With the quick increment in the number of vehicles and the savagely blocked Indian roads, road safety has turned into a factor of most extreme significance for the nation's residents. Accidents on the street have become a significant concern for individuals as well as the government. In 2018, there were around 151 thousand deaths due to road accidents in India [2]. Therefore in India, the surveillance system and intelligent traffic management are the essential requirements for smart city improvement. A Smart traffic checking system is not completed without the presence of a system that is competent to recognize any traffic issues consequently. Traffic jams can be identified from vehicle speed that is moderate and also a high volume of vehicles so that it is critical to identify and appraise vehicle speed for realizing street profile dependent on the vehicle speed. Also, there are numerous explores for counting and classifying vehicle objects from video images in which including estimation of vehicle speed procedure that will commit to constructing a smart system for traffic [3]. So that traffic management authority needs a better surveillance system. Due to the significant expense of radar and less precision, the radar system is not able to become popular in the traffic surveillance system. And the inductive loops technique also needs high support. Therefore it is necessary to propose some mechanisms to overcome such problems.

This paper proposed a novel computer vision-based automated system for multi-vehicle detection, tracking, and estimation of vehicle speed in a video sequence by using image processing. The main contributions of the proposed system are:

-To develop a method for multi-vehicle detection.

-To implement a technique for vehicle tracking.

-To implement a technique for vehicle speed estimation of moving single or multi vehicle in video sequence using image processing.

\section{RELATED WORK}

Techniques of image processing had been applied extensively in various fields. Efforts have been made by various researchers to adopt image processing techniques for estimation of vehicles speed are discussed below.

Alexander A S Gunawana et al., (2019) proposed a Direct Linear Transformation (DLT) method for road image projection, Background Subtraction method is used for vehicle position recognition and tracked utilizing Mixture of Gaussian (MoG) to evaluate the vehicle speed. The Euclidean distance method is used. Vehicle position identification with groundtruth is 12.07 pixels with a camera point of $40^{\circ}$. But it cannot be applied for multi-vehicle detection. And vehicle detection is not influenced by the vehicle shadow [4].

Danang Wahyu Wicaksono et al., (2017) implemented the Euclidean distance strategy with a wide range of camera plots for vehicle speed estimation. This system is fit on evaluating the moving vehicle speed. But the highest error for moving vehicle speed estimation in this method depends on vehicle speed estimation on high-speed vehicles and two objects are close to one another will be identified as one vehicle. [5]. Diogo C et al., (2017) developed the work on vehicle speed estimation, tracking of different features and selection located 
within every region of the vehicle's license plate. This proposed system was related to the ground truth of speeds which is acquired by inductive loop detectors and also physically labeled ground truth regions of license plate. The system used a license plate recognition and blob tracking algorithm. But all vehicles not contain visible license plate and the ground truth speed sometimes which is not properly mentioned a speed to a vehicle [6].

Suresh et al., (2016) implemented a method on the Estimation of Moving Vehicle Speed using Image Processing. This strategy is used for calculating vehicle speed by tracking the movement of the vehicle. The speed determination is done by using length traveled by the vehicle over frame rate and the number of frames. But this proposed work not handles multivehicle detection at a time; the camera angle is not determined [7].

Jinhui Lan et al., (2014) implemented a vehicle speed estimation technique which used three frames differencing strategies and an optical flow method to estimate vehicle speed and recommended also a strategy to calculate the velocity of vehicle dependent on mono camera using a threeframe difference method to extract moving vehicles contours properly. But they calculated optical flow values only in shapes pixels to get vehicle speed in pixels/sec then in $\mathrm{Km} / \mathrm{hr}$, the optical flow algorithm is more sensitive to noise, has high complexity algorithm [8]. Asif Khan et al., (2014) proposed the Euclidean distance method to estimate vehicle speed using the image processing method. But this method didn't explore ROI choice and the impact of camera angles for the accurate outcome for estimation of vehicle speed from video data [9]. Leite A.V. et al., (2014) proposed a method to achieve speed estimation in induction motor sensor less control. The vehicle speed estimation method is based on a reduced order extended Kalman filter. This algorithm uses a reduced order state space system structure that is discretized in a specific and creative manner proposed. Rotor motion segments are detected rather than the rotor speed itself. But this system also requires high maintenance and installation cost, also it does not provide enough data about traffic related parameters [10].

Kassen N. et al., (2012) proposed an improved method by using the Random forest method for vehicle speed estimation. But it will give high computational expenses and utilizes more memory, slow prediction, which is a difficulty to applications [11]. Shedbalkar K. et al., (2012) implemented a vehicle speed detection method for PMSM which is based on an extended $\mathrm{KF}$ algorithm. Vehicle speed estimation is necessary for Sensorless vector control of the machine. This system is intended to estimate the speed of rotors as per the mathematical equation. But Permanent magnet synchronous motors require a driver, needs a complex control system including power electronics and microcontrollers and needs a higher initial cost, difficult to start up [12].

Dogan et al., (2010) proposed the Lucas-Kanade optical flow algorithm for vehicle tracking. Estimated vehicle speed by tracking and calculating velocity vectors of a set of reference points by using a side view mono camera that distinguished on the vehicle under daytime conditions. Vamsi Krishna et al., (2010) implemented vehicle detection, speed estimation of vehicle technique. The movement is derived in using a spherical projection equation to estimate the vehicle speed. For movement tracking used Lucas Kanade Tomasi method. But these algorithms can handle only a single vehicle at once [13, 14]. Rad et al., (2010) proposed a vehicle speed estimation method that consists of CVS as a background method and Euclidean distance and accuracy 90\%. In this study mean filter method is used for background generation. But the mean is a less robust [15].

\section{MATERIALS AND METHODS}

The main aim of the proposed system is to design and development of an efficient approach for vehicle detection, vehicle tracking, and vehicle speed estimation of moving single or multi-vehicle in a video sequence using image processing. To achieve the purposes of this proposed system, a methodological structure is developed (see Fig. 1). This proposed system is broadly divided into 3 stages. The first stage is the detection of multi-vehicle and then track that detected vehicle and at the last estimation of vehicle speed.

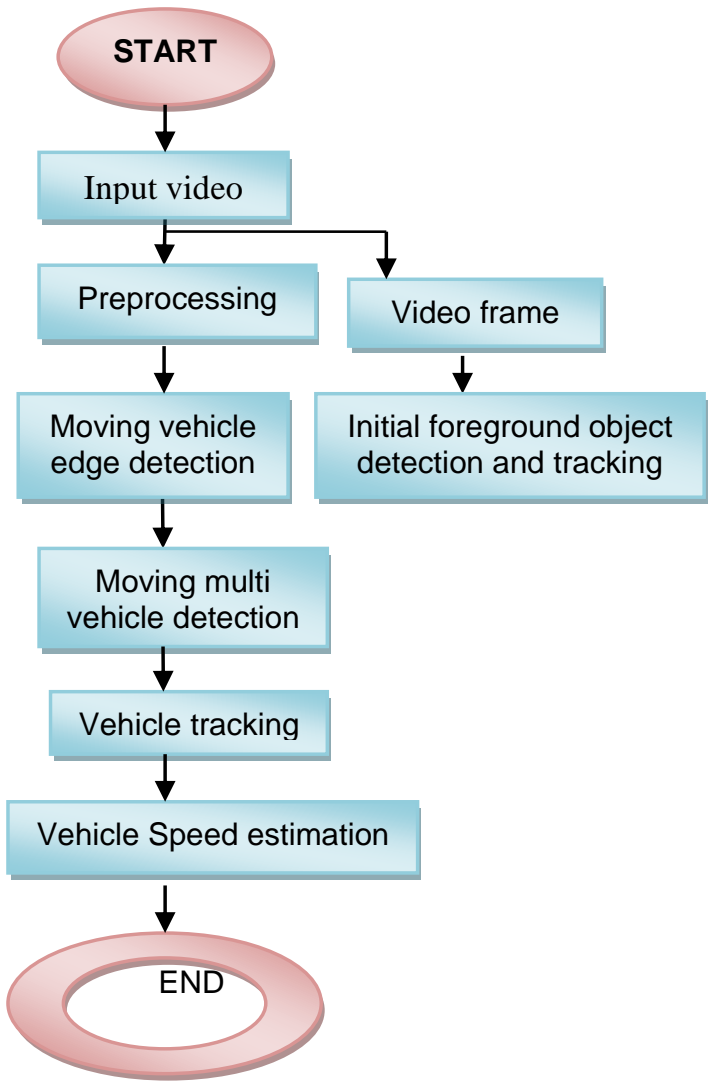

Fig.1. Flow chart of proposed system

\section{A. Detection of Single or Multi-vehicle}

The first step of the proposed system is to perform moving multi-vehicle detection using a gradient edge detection algorithm and normal cross-correlation along with used background subtraction algorithm for initial object detection. Before vehicle detection preprocessing will takes place.

(i) Preprocessing: The median filter algorithm is used to eliminate noise from the video image frame. The reduction of noise from the video is one of the pre-processing steps to improve the outcome of further processing methods. The median is a more robust method and much better in sharp edges preserving compared to the mean filter method [16]. 
Algorithm: Median Filtering Algorithm for Image Noise Reduction

Input: Video frames

Output: Noise removed frames

Step1: Taking whole video frames as an input.

Step2: Median filter method is applied to remove unwanted noise from the video image frame.

Step3: Applied filtering process at each image frame pixel by pixel that is as shown in below form:

$$
\mathrm{Y}[\mathrm{r}, \mathrm{s}]=\operatorname{median}\{\mathrm{X}[\mathrm{i}, \mathrm{j}],(\mathrm{i}, \mathrm{j})\}
$$

Where, $X[i, j],(i, j)$ is a input image ,centered around location $[r, s]$ in the image.

Step4: Produced filtered video frame which is not contains any noise.

(ii) Vehicle Edge Detection algorithm: Gradient edge detection algorithm which detects each vehicle edges by taking the maximum and minimum values in the first derivative of the image frame. In this proposed work we find out gradient image frame. The first-order derivative of Image frame $I$ in the $\mathrm{X}$-direction and the y-direction. As shown in Fig.2.

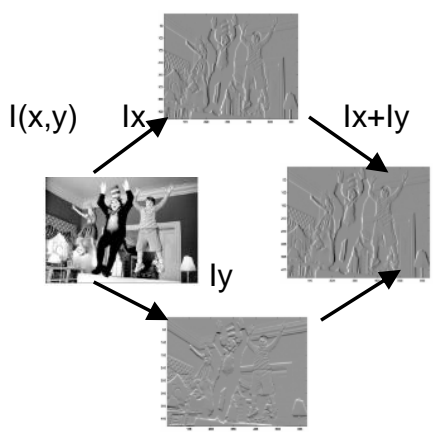

Fig.2. Edge detection using Gradients

Algorithm: Gradient edge detection algorithm for vehicle edge detection

Input: Input video frames

Output: Gradient edge detected video frames

Step1: Input video frames $\mathrm{I}(\mathrm{x}, \mathrm{y})$ is taken as input and produces (Ix, Iy) i. e (Ix+Iy)

$$
\begin{aligned}
& I(x, y)=I x \\
& I(x, y)=I y
\end{aligned}
$$

Step2: Compute an image gradient vector at each and every pixel by convolving image frame with vertical derivative filters and horizontal derivative filters.

$$
\begin{aligned}
& \mathrm{Ix}=\mathrm{dI}(\mathrm{x}, \mathrm{y}) / \mathrm{dx} \\
& \mathrm{Iy}=\mathrm{dI}(\mathrm{x}, \mathrm{y}) / \mathrm{dy}
\end{aligned}
$$

Where $\mathrm{dI}(\mathrm{x}, \mathrm{y}) / \mathrm{dx}$ and $\mathrm{dI}(\mathrm{x}, \mathrm{y}) / \mathrm{dy}$ are the first order derivative of Image I in $\mathrm{x}$ (horizontal) and in y (vertical) direction.

Step3: Displayed gradient image. (iii) Background subtraction for object detection: Integrated background subtraction algorithm for moving object detection using the initial frame. Detect and count the vehicle in a video image sequence using the foreground detector method based on the Gaussian mixture system method. This algorithm shows a foreground detector, count vehicle and blob analysis to detect the vehicle in a video image sequence. This algorithm needs a static camera.

Algorithm: Moving object detection using background subtraction algorithm

Input: Initial frame extracted from input video

Output: Detected vehicle frame

Step1: read all input video frames.

Step2: Identify the foreground in the current video frame.

Step3: Apply the morphological opening to eliminate noise in the foreground.

Step4: Detect the connected elements with the defined minimum area and measure their bounding boxes.

Step5: Draw bounding boxes throughout the detected cars.

Step6: Display the number of cars detected in the video frame.

Step7: visualize the results.

(iv)Vehicle detection using Normalized cross correlation algorithm: To evaluate the similarity or dissimilarity degree between two compared images used normalized crosscorrelation algorithm as a metric. The main purpose of NCC is to identify the areas which contain a vehicle in the image. The cross-correlation is mainly used for this purpose which is a good method because it is easily estimated the degree of similarity, which can also tell like that to estimate how much two image frames are correlated [17].

Algorithm: Template matching in vehicle detection using Normalized cross correlation algorithm.

Input: Input template, input image

Output: Correlation coefficients

Step1: Input image $p(i, j)$ and input template $(i, j)$ is taken as input and produces correlation coefficient $\rho$ :

$$
\rho=\sum_{i, j} \frac{\left(p\left(i_{i}, \hat{j}\right)-\bar{p}\right)\left(q\left(i_{i} \hat{j}\right)-\bar{q}\right)}{\sigma p \sigma q}
$$

Where $p\left(i_{r} j\right)$ is the input image which is shared by template and $\bar{p}$ is the mean of $p\left(i_{i} j\right) ; q\left(i_{i} j\right)$ is the template and $\bar{q}$ is the mean of $q\left(i_{i} j\right)$; $\sigma p$ and $\sigma q$ are the standard deviations of $p\left(i_{i} j\right)$ and $q\left(i_{i} j\right)$ respectively.

Step2:Compute the normalized cross-correlation coefficient $\rho$. The peak of the cross-correlation matrix occurs where the sub_images are best correlated. It works on only grayscale images.

Step3: Find the Total Offset between the Images and peak in cross-correlation.

Step4: After getting $\mathrm{x}$ and $\mathrm{y}$ axis peak value computed offset between the images. 
Step5: The matched area. That is $\mathrm{x}, \mathrm{y}$ Width, and height of the ROI as the 4-element vector.

\section{B. Vehicle Tracking}

In moving vehicle tracking the feature extraction is the main key aspect. The various methods of feature extraction are available in the existing methods. Features are nothing but some of the detected vehicle characteristics. The second step of the proposed system is to track the vehicle using current and previous frame coordinates. After template matching algorithm, coordinates are output, using that coordinates initialized tracker and pick bounding box in the format $[\mathrm{x}, \mathrm{y}$, $\mathrm{w}, \mathrm{h}]$. The extracted vehicle characters are tracked of all video image sequential frames.

\section{Algorithm: Vehicle tracking using tracking algorithm}

Input: Current frame and previous frame from video frame

Output: Tracked moving vehicles

Step1: Previous and current frames taken as input and produces tracking vehicle.

Step2: Initialized previous vehicle length as a vehicle tracker coordinate.

Step3: Computed old and new frame tracker.

Step4: Old and new frame is converted RGB to gray image. Put boding box to tracker.

Step5: Compute gradient products of derivatives at every pixel, formula given below:

$$
\begin{aligned}
& \mathrm{Ix} 2=\mathrm{Ix} \wedge 2 \\
& \text { IxIy }=\mathrm{Ix} \times \mathrm{Iy} \\
& \mathrm{Iy} 2=\mathrm{Iy} \wedge 2
\end{aligned}
$$

Step6: Define sum of each pixel the matrix.

Step7: If ' $i$ ' is equal to initial frame +1 then it will get current frame then result of frame is calculated.

Step4: If current and previous frame tracer coordinated not matched, drawn bounding box around detected vehicle and track that vehicles.

\section{Vehicle Speed Estimation:}

The vehicle speed estimation process is the final step of the proposed system. The vehicle speed in each image frame is estimated by using the vehicle position in each image frame. The frame rate of the captured video dataset is a known value, for that reason the estimation of vehicle speed becomes possible. To find out the distance traveled by the pixel in each frame, if the pixel has the coordinate that is $\mathrm{k}=(\mathrm{p}, \mathrm{q})$ and $\mathrm{k}$ $1=(r, s)$. Where the location is showed in frame $\mathrm{k}$ and $\mathrm{k}-1$ for one vehicle with $(\mathrm{p}, \mathrm{q})$ and $(\mathrm{r}, \mathrm{s})$ coordinate.

\section{Algorithm: Vehicle speed estimation}

Input: current and previous frame length, template and input video frame rate

Output: Estimated vehicle speed.

Step1: Frame rate and difference between two frames is taken as input and average speed is produced as shown in the below equation:

$$
\text { Speed }=\text { mean }(\text { B) } /(\text { D_Ratio })
$$

Where, $\mathrm{B}$ is the product of frame distance and frame rate, D_Ratio is the trained vehicle.

Step3: Variable B is represented as product of frame rate and vehicle frame difference as shown in the below equation:

$$
\mathrm{B}=\mathrm{V} \_ \text {diff } \times \text { FrameRate }
$$

Where, V_diif is the length of two frames, FrameRate is a frame per pixel.

Step4: Length of two frames is represented as distance between two frame coordinated, as shown in the below form:

$$
\text { V_diff }=\operatorname{coord}(1)-\operatorname{coord}(2)
$$

\section{RESULTS AND DISCUSSION}

The dataset used for implementation is Landzs/Tracking Multiple_objects_In Surveillance_Cameras/tree/master/Data dataset [18]. This dataset contains a different type of sample videos. Videos are in .avi / .MP4 / .mat format. These videos are captured from the Surveillance camera. The frame rate of the used dataset is $29.97 \mathrm{fps}$. The video is displayed around $10 \mathrm{sec}$ which contains 270 frames. The used dataset consists of $640 \times 480$ pixels. It should be evaluated qualitatively by the visual result and quantitatively by measuring metrics.

\section{A. Visual Results}

Here we considered 3 different sample video datasets. The visual result of dataset 1 for vehicle speed estimation is shown in the below figures. The captured video is pre-processed to get high accuracy. The pre-processed result figure is displayed in the output and Vehicle edge detection is done by using a gradient edge detection algorithm. The result of gradient edge detection is shown in Fig.3. The result of the background subtraction method as shown in fig.4. (a) initial video frame, (b) foreground frame, (c) morphological operation, (d) Initial foreground detected frame. Based on vehicle features vehicle tracking is done. In the proposed system used vehicle edges as features, based on that vehicle tracking is carried out. The frame matching algorithm is support for vehicle tracking. The result of both moving vehicles detection and tracking is shown in Fig.5. The final work of the proposed system is to estimate vehicle speed. In this proposed system, calculated vehicle speed using frames and frame rate. The result of the system detected speed and actual speed as shown in Fig.6.

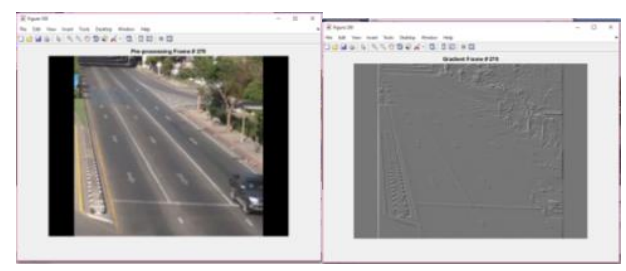

(a)

(b)

Fig.3. Results of preprocessing and gradient edge detection method (a) Preprocessing frame (b) Gradient frame 


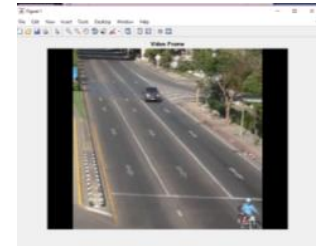

(a)

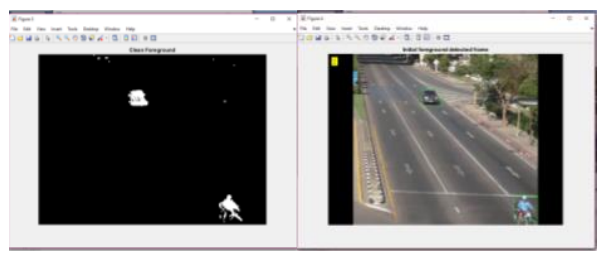

(c)

(d)

Fig.4. Results of background subtraction method: (a) Video Frame (b) Foreground frame (c) Morphological Operation (d) Initial foreground Detected frame

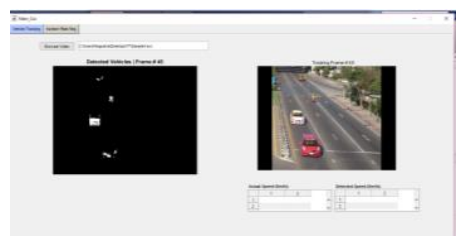

Fig.5. left side moving multi-vehicle detection and right side multi-vehicle tracking

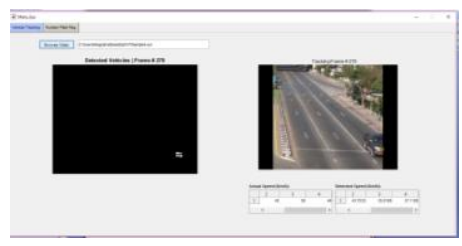

Fig.6. vehicle speed estimation

\section{B. Performance Measurements}

(i)Accuracy: Accuracy is an important performance measure and it is simply a ratio of correctly predicted observation to the total observation. Accuracy is the level of prediction that is nearest to the actual label. A higher value of accuracy denotes better performance which is given by the below equation [19].

Accuracy $=(T P+T N) /(T P+T N+F P+F N)$

Where: $\mathrm{TN}=$ True negative $; \mathrm{TP}=$ True positive $; \mathrm{FN}=$ False negative; $\mathrm{FP}=$ False positive

(ii) Misclassification Rate (Error rate): The error rate is equivalent to one minus accuracy value [20] as shown in the below equation:

$$
\text { Error rate }=1-\text { Accuracy }
$$

(iii)Sensitivity (Recall or True positive rate): Sensitivity is nothing but the ratio of the number of vehicles correctly detected to the actual number of vehicles [21].

$$
T P R=T P / P=T P /(T P+F N)
$$

Where, TP is true-positive and FN is false-negative
(iv)Specificity: Specificity or true negative rate is the ratio of the number of vehicles not detected to the actual number of the vehicle not in the scene [20]:

$$
T N R=T N / N=1
$$

Where, TN true negative, FP is false positive

(v)Precision (Positive predictive value): The precision is the ratio of true positive condition with the total number of true positive and false positive condition. This comprises of the TP and false positive (FP) detections [22].

$$
P P V=T P /(T P+F P)
$$

Where, TP is true-positive, FP is false-positive

(vi)FPR-False positive rate (Fall-out): The false positive rate is the ratio of vehicle declaration in predicted condition to the actual number of vehicles not in the scene [20].

$$
F P R=F P / N=F P /(F P+T N)
$$

(vii)F1_score: F1_score is a weighted average of the true positive rate or recall and precision. Therefore, this score takes both false positive and false negative into account. F1 is more useful than accuracy. The equation of F1_score is given below:

$$
F 1=2 \times(P P V \times T P R / P P V+T P R)=2 T P /(2 T P+F P+
$$$$
F N \text { ) }
$$

Where PPV is positive predictive value, TPR is true positive rate

(viii)MCC-Matthews correlation coefficient: $\mathrm{MCC}$ is in essence a correlation coefficient between the observed and predicted binary classifications; it returns a value between -1 and +1 . A coefficient of +1 is the perfect prediction in vehicle detection, 0 no better than random prediction and -1 is a total disagreement between prediction and observation. The MCC can be calculated directly from the confusion matrix using the formula [23]:

$$
\frac{T P \times T N-F P \times F N}{\sqrt{(T P+F P)(T P+F N)(T N+F P)(T N+F N)}}
$$

Where TP is true positives, TN is true negatives, FP is false positives and $\mathrm{FN}$ is false negatives. If any of the four sums in the denominator is zero, the denominator can be arbitrarily set to one.

(ix)kappa-Cohen's kappa: This is essentially a measure of how well the classifier performed as compared to how well it would have performed simply by chance. In other words, a system will have a high Kappa score if there is a big difference between the accuracy and the null error rate [20].

$$
K=p_{0}-p e / 1-p e=p e-p e
$$

Where; po = vehicle detection accuracy

$$
\begin{gathered}
P e=((P \times(T P+F P))+(N \times(F N+T N))) /((T P+T N+ \\
\left.F P+F N)^{\wedge} 2\right)
\end{gathered}
$$

po is the relative observed detected vehicle (identical to accuracy), pe is the hypothetical probability of chance detect vehicle, using the observed data to calculate the probabilities of each observer randomly seeing each category. 


\section{Quantitative Analysis}

The performance of the proposed method has been evaluated and the parameters have been tabulated in the following tables. The proposed methodology involves more efficient methods for vehicle speed estimation. These algorithms produced good performance results compared to other methods. Hence, the performance of the proposed system can be measured using various metrics such as accuracy, FPR, error rate, sensitivity, etc given in Table 1. Vehicle detection and tracking performance are represented graphically through a performance graph. The accuracy level gained for vehicle detection and tracking is represented in Fig.7.The accuracy level gained by accuracy and kappa for overall vehicle detection and tracking is represented in Fig.8. The performance rate gained by sensitivity, specificity, F1_score, precision, MCC for vehicle detection and tracking is represented in the Fig.9. The error rate gained by error and FPR for detection and tracking of vehicles is represented in Fig. 10.

Table 1: Performance result of vehicle detection and tracking.

\begin{tabular}{|c|c|}
\hline Parameters & Sample4.avi \\
\hline TP & 379 \\
\hline TN & 351 \\
\hline FP & 7 \\
\hline FN & 0.9733 \\
\hline Detection Accuracy & 0.972 \\
\hline Tracking Accuracy & 0.0267 \\
\hline Error & 0.0251 \\
\hline False Positive Rate & 0.9668 \\
\hline Sensitivity & 0.9804 \\
\hline Specificity & 0.9819 \\
\hline Precision & 0.9743 \\
\hline F1_score & 0.9467 \\
\hline $\begin{array}{c}\text { Matthews Correlation } \\
\text { Coefficient (MCC) }\end{array}$ & 0.9466 \\
\hline Kappa
\end{tabular}

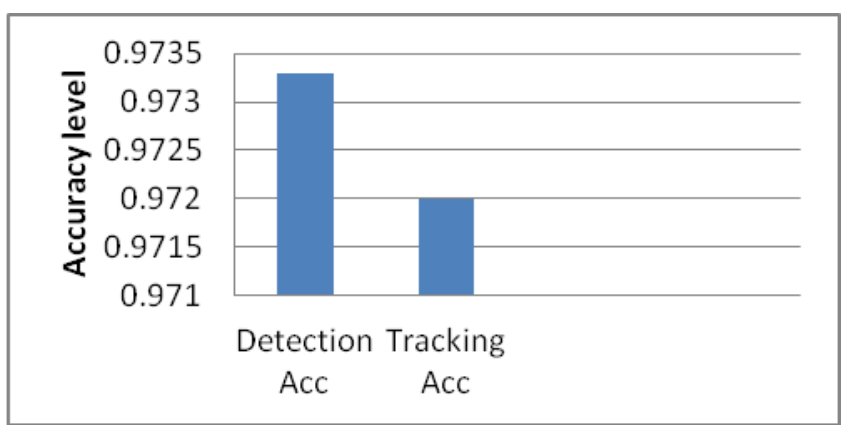

Fig.7. Vehicle detection and tracking accuracy measure graph

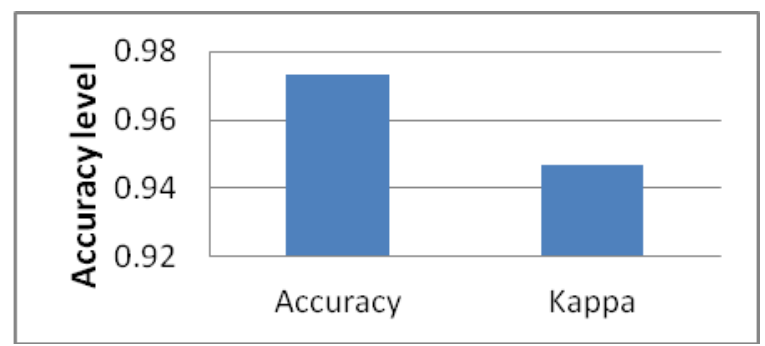

Fig.8. Accuracy and kappa measure graph

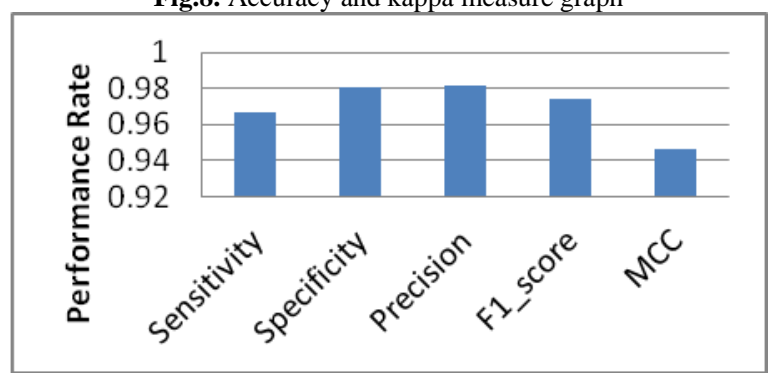

Fig.9. Sensitivity, specificity, precision, F1_score, MCC measure graph

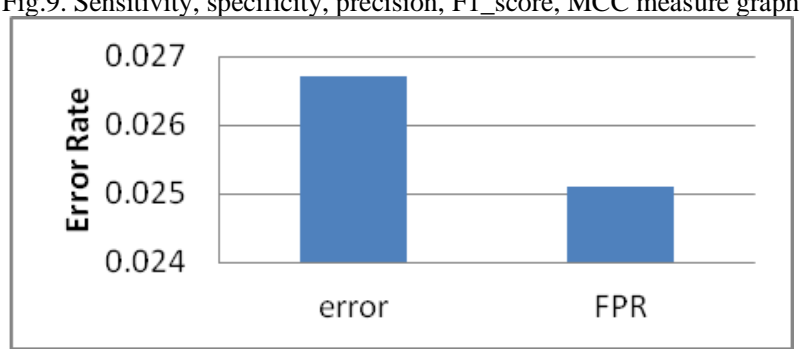

Fig.10. Error and FPR measure graph Measure graph

Table 2 shows the performance evaluation of vehicle speed measurements in the proposed method at a frame rate of $29.97 \mathrm{fps}$. The first column 1 of the table gives the number of the vehicle. Column 2 shows the frame rate of sample video, column 3 is the actual vehicle speed in kilometer per hour, column 4 and 5 is system detected speed $(\mathrm{k} / \mathrm{h})$ and column 6 is the error of speed calculation. The average system detected vehicle speed is calculated by using frame rate, length of frames.

Table 2.The performance evaluation of vehicle speed measurement in the proposed method at a frame rate $=29.97 \mathrm{fps}$

\begin{tabular}{|c|c|c|c|c|c|}
\hline Vehicle no. & $\begin{array}{c}\text { FrameRat } \\
\mathrm{e}\end{array}$ & $\begin{array}{c}\text { Actual } \\
\text { speed(k/h) }\end{array}$ & $\begin{array}{c}\text { Detected } \\
\text { speed(m/s) }\end{array}$ & $\begin{array}{c}\text { Detected } \\
\text { speed(k/h) }\end{array}$ & Error \\
\hline V1 & 29.97 & 45 & 12.19178 & 43.8904 & -1.1096 \\
\hline V2 & 29.97 & 45 & 12.15369 & 43.7533 & -1.2467 \\
\hline V3 & 29.97 & 50 & 15.28319 & 55.0195 & +5.0195 \\
\hline V4 & 29.97 & 40 & 10.30847 & 37.1105 & -2.8895 \\
\hline
\end{tabular}

Table 3 shows the vehicle detection and tracking performance comparison between three different samples that is Sample 1, Sample 2 and Sample 4. The accuracy gained for three different samples is shown in Fig.11.The error rate is gained for three samples are represented in Fig.12. The performance rate is for three samples are given in Fig.13. Table 4 and Table 5 is the performance evaluation of vehicle speed measurement in the proposed method at a frame rate $=29.97 \mathrm{fps}$. In that first column is vehicle number and the next one is the frame rate, 3rd is actual speed, 4th is detected speed and the last one is error 
Table 3 Performance comparison using different samples

\begin{tabular}{|c|c|c|c|}
\hline \multirow{2}{*}{ Parameters } & \multicolumn{3}{|c|}{ Videos } \\
\cline { 2 - 4 } & Sample1 & Sample2 & Sample4 \\
\hline Detection Accuracy & 0.9789 & 0.9945 & 0.9733 \\
\hline Tracking Accuracy & 0.978 & 0.986 & 0.972 \\
\hline Error & 0.0211 & 0.0055 & 0.0267 \\
\hline False Positive Rate & 0.0455 & 0.0138 & 0.0251 \\
\hline Sensitivity & 1 & 1 & 0.9668 \\
\hline Specificity & 0.9545 & 0.9862 & 0.9804 \\
\hline Precision & 0.9623 & 0.991 & 0.9819 \\
\hline F1_score & 0.9808 & 0.9955 & 0.9743 \\
\hline Kappa & 0.9584 & 0.9886 & 0.9467 \\
\hline
\end{tabular}

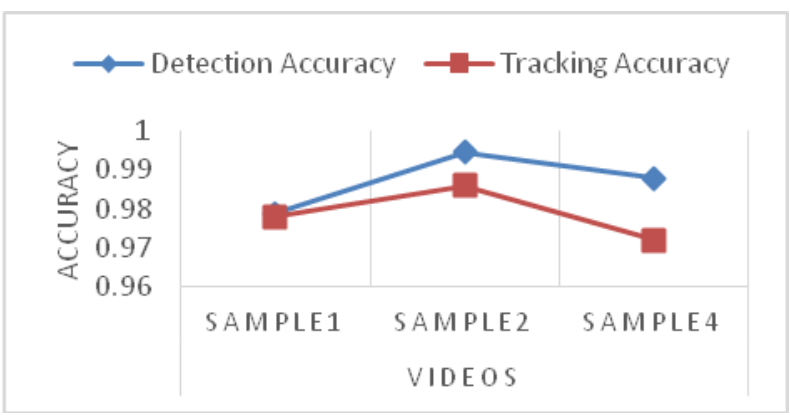

Fig.11. Accuracy analysis graph

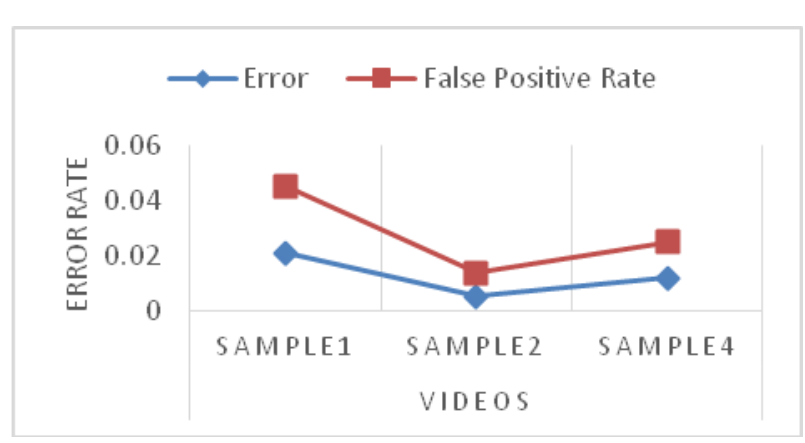

Fig.12.Error rate for different type of video samples graph

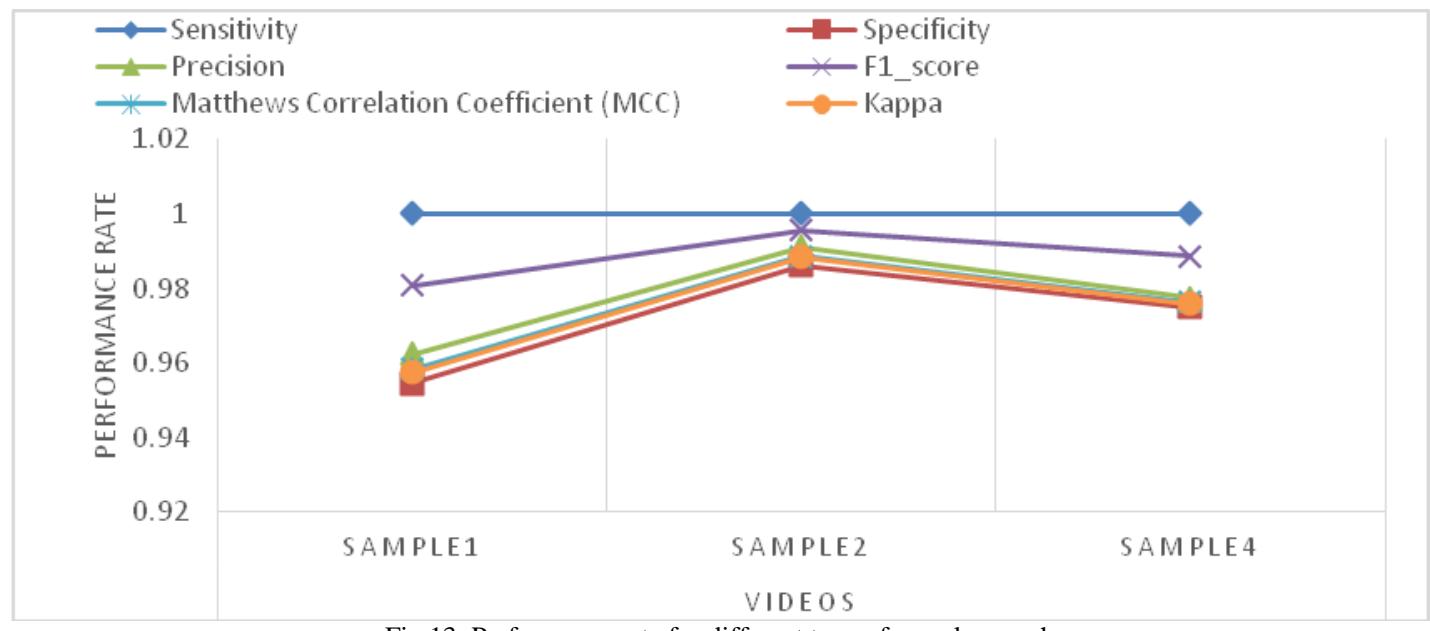

Fig.13. Performance rate for different type of samples graph

Table 4.The performance evaluation of vehicle speed measurement in the proposed method at a frame rate $=29.97 \mathrm{fps}($ Sample1)

\begin{tabular}{|c|c|c|c|c|c|}
\hline $\begin{array}{c}\text { Vehicle } \\
\text { no. }\end{array}$ & $\begin{array}{c}\text { FrameRat } \\
\mathrm{e}\end{array}$ & $\begin{array}{c}\text { Actual } \\
\text { speed }(\mathrm{k} / \mathrm{h})\end{array}$ & Detected speed $(\mathrm{m} / \mathrm{s})$ & Detected speed(k/h) & Error \\
\hline V1 & 29.97 & 45 & 12.19178 & 43.8904 & -1.1096 \\
\hline
\end{tabular}

Table 5.The performance evaluation of vehicle speed measurement in the proposed method at a frame rate $=29.97 \mathrm{fps}($ Sample2)

\begin{tabular}{|c|c|c|c|c|c|}
\hline $\begin{array}{c}\text { Vehicle } \\
\text { no. }\end{array}$ & $\begin{array}{c}\text { FrameRat } \\
\mathrm{e}\end{array}$ & $\begin{array}{c}\text { Actual } \\
\text { speed }(\mathrm{k} / \mathrm{h})\end{array}$ & Detected speed $(\mathrm{m} / \mathrm{s})$ & Detected speed $(\mathrm{k} / \mathrm{h})$ & Error \\
\hline V1 & 29.97 & 45 & 12.19178 & 43.8904 & -1.1096 \\
\hline
\end{tabular}

\section{V.CONCLUSION}

The proposed methodology involves the vehicle speed estimation which is done by using the image processing technique. Vehicle speed estimation technique is carried out using different approaches that is moving multi-vehicle detection, tracking that detected vehicle which results in higher accuracy. The experiment was carried out on 3 different video samples of the .mat lavilmp4 file format. The multi-vehicles are detected by the gradient edge detection and normal cross correlation algorithm. Further, these detected vehicles are tracked using a two frame difference algorithm. Finally, the vehicle speed is estimated by using tracked vehicle features difference distance and frame rate. The 
performance of the proposed system is measured by four accuracy measures namely Accuracy, error, false positive rate, Precision, F1_score, MCC, Kappa, sensitivity, specificity. The vehicle detection and tracking accuracy value for the proposed system is $97.33 \%, 97.2 \%$. Error rate value obtained is 0.0267 , FPR value is 0.0251 , Sensitivity value is $96.68 \%$, Specificity value is $98.04 \%$, Precision value is $98.19 \%, F 1 \_s c o r e$ value is $97.43 \%, \mathrm{MCC}$ value is 0.9467 and the kappa for the proposed system is 0.9466 . Hence, it shows that the proposed method has high accuracy than the existing system. Finally, it can be concluded that speed estimation through video image processing is more efficient and economical than radar technology.

\section{FUTURE SCOPE}

In future work the support vector machine algorithm could be adapted for the vehicle speed estimation because it gives good estimation result, Due to the low resolution of the video dataset number plate is not detected properly so future work is to collect high resolution video dataset which helps for detecting vehicle number plate properly and execution speed of the algorithms used is a crucial measure to be taken into account while developing vehicle speed estimation architecture.

\section{REFERENCES}

[1] https://www.statista.com/statistics/269872/largest-automobilemarkets-worldwide-based-on-new-car-registrations/

[2] https://www.statista.com/statistics/746887/india-number-of-fatalitiesin-road-accidents/

[3] B. C. Putra. (2016). Moving vehicle classification with fuzzy logic based on image processing. Master's thesis, Sepuluh Nopembe Institute of Technology.

[4] Alexander A S Gunawan, Deasy Aprilia Tanjung, Fergyanto E. Gunawan.(2019).Detection of Vehicle Position and Speed using Camera Calibration and Image Projection Methods. Procedia Computer Science, Vol.157,255-265.

[5] Danang Wahyu Wicaksono and Budi Setiyono. (2017).Speed Estimation on Moving Vehicle Based on Digital Image Processing. International journal of computing science and applied mathematics, Vol.3(1): 1-21

[6] D. C. Luvizon, B. T. Nassu and R. Minetto.(2017).A Video-Based System for Vehicle Speed Measurement in Urban Roadways. IEEE Transactions on Intelligent Transportation Systems, Vol.18(6):13931404

[7] B. Suresh, K. Triveni, Y. V. Lakshmi, P. Saritha, K. Sriharsha, D. Srinivas Reddy.(2016).Determination of Moving Vehicle Speed using Image Processing. International journal of engineering research \& technology (ijert) ncacspv, Vol.4, pp.1-4.
[8] J. Lan, J. Li, G. Hu, B. Ran, and L. Wang.(2014).Vehicle speed measurement based on gray constraint optical flow algorithm. Optik International Journal for Light and Electron Optics, Vol.125(1):289295.

[9] A. Khan, I. Ansari, M. S. Z. Sarker, and S. Rayamajhi.(2014).Speed estimation of vehicle in intelligent traffic surveillance system using video image processing. International Journal of Scientific \& Engineering Research, Vol. 5(12):1384-1390.

[10] A. V. Leite, R. E. Araujo and D. Freitas.(2014).A new approach for speed estimation in induction motor drives based on a reduced-order extended Kalman filter. IEEE International Symposium on Industrial Electronics, Ajaccio, France, Vol. 2, 1221-1226.

[11] N. Kassem, A. E. Kosba and M. Youssef.(2012).RF-Based Vehicle Detection and Speed Estimation. IEEE 75th Vehicular Technology Conference (VTC Spring), Yokohama,1-5.

[12] K. Shedbalkar, A. P. Dhamangaonkar and A. B. P. Walchand.(2012).Speed estimation using extended Kalman filter for PMSM. International Conference on Emerging Trends in Electrical Engineering and Energy Management (ICETEEEM), Chennai,433 435 .

[13] Dogan, Sedat \& Temiz, Mahir \& Külür, Sitk1.(2010).Real Time Speed Estimation of Moving Vehicles from Side View Images from an Uncalibrated Video Camera. Multidisciplinary Digital Publishing Institute (MDPI), Sensors (Basel, Switzerland), Vol.10(5):4805-24.

[14] V. K. Madasu and M. Hanmandlu.(2010). Estimation of vehicle speed by motion tracking on image sequences.IEEE Intelligent Vehicles Symposium, San Diego, CA,185-190.

[15] Gholami, Arash \& Dehghani, Abbas \& Karim, Mohamed.(2010). Vehicle speed detection in video image sequences using CVS method. International Journal of Physical Sciences, Vol 5(17):2555-2563.

[16] https://homepages.inf.ed.ac.uk/rbf/HIPR2/median.htm

[17] S.-D. Wei and S.-H. Lai. (2008). Fast template matching based on normalized cross correlation with adaptive multilevel winner update. IEEE Transactions on Image Processing, Vol. 17(11): 2227-2235.

[18] https://github.com/Landzs/Tracking_Multiple_Objects_In_Surveillan ce Cameras/tree/master/Data

[19] Sundoro, H.S. \& Harjoko, Agus. (2016). Vehicle counting and vehicle speed measurement based on video processing. Journal of Theoretical and Applied Information Technology, Vol. 84(2):233241.

[20] K. Qian. (2014). Simple guide to confusion matrix terminology. Data School.

[21] Hanif, Adnan \& Mansoor, Atif \& Imran, Ali. (2018).Performance Analysis of Vehicle Detection Techniques: A Concise Survey.World Conference on Information Systems and Technologies, Vol. 746, 491-500.

[22] Billones, Robert Kerwin \& Bandala, Argel \& Gan Lim, Laurence \& Sybingco, Edwin \& Fillone, Alexis \& Dadios, Elmer. (2018). Microscopic Road Traffic Scene Analysis Using Computer Vision and Traffic Flow Modelling. Journal of Advanced Computationa Intelligence and Intelligent Informatics, Vol. 22, 704-710.

[23] Boughorbel, S.B. (2017). Optimal classifier for imbalanced data using Matthews Correlation Coefficient metric. PLOS ONE, Vol.12 (6):1- 